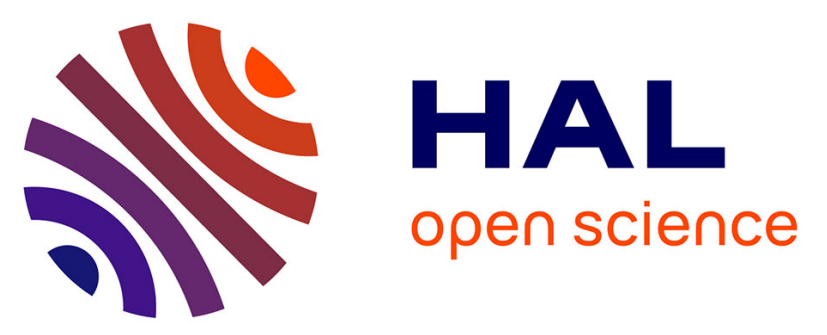

\title{
Analysis of Cardiac Micro-Acceleration Signals for the Estimation of Systolic and Diastolic Time Intervals in Cardiac Resynchronization Therapy
}

Lionel Giorgis, Alfredo I. Hernandez, Amel Amblard, Lotfi Senhadji, Serge Cazeau, Gaël Jauvert, Erwan Donal

\section{To cite this version:}

Lionel Giorgis, Alfredo I. Hernandez, Amel Amblard, Lotfi Senhadji, Serge Cazeau, et al.. Analysis of Cardiac Micro-Acceleration Signals for the Estimation of Systolic and Diastolic Time Intervals in Cardiac Resynchronization Therapy. Computers In Cardiology, Sep 2008, Bologna, Italy. pp.393-396. inserm-00349229

\section{HAL Id: inserm-00349229 https://www.hal.inserm.fr/inserm-00349229}

Submitted on 25 Dec 2008

HAL is a multi-disciplinary open access archive for the deposit and dissemination of scientific research documents, whether they are published or not. The documents may come from teaching and research institutions in France or abroad, or from public or private research centers.
L'archive ouverte pluridisciplinaire HAL, est destinée au dépôt et à la diffusion de documents scientifiques de niveau recherche, publiés ou non, émanant des établissements d'enseignement et de recherche français ou étrangers, des laboratoires publics ou privés. 


\title{
Analysis of Cardiac Micro-Acceleration Signals for the Estimation of Systolic and Diastolic Time Intervals in Cardiac Resynchronization Therapy
}

\author{
L Giorgis $^{1}$, AI Hernandez ${ }^{2,3}$, A Amblard ${ }^{1}$, L Senhadji $^{2,3}$, S Cazeau $^{4}$, G Jauvert ${ }^{4}$, E Donal $^{2,3,5}$ \\ ${ }^{1}$ Sorin Group ELA Medical, Le Plessis-Robinson, F-92357, France \\ ${ }^{2}$ INSERM, U642, Rennes, F-35000, France \\ ${ }^{3}$ Université de Rennes 1, LTSI, Rennes, F-35000, France \\ ${ }^{4}$ Groupe Inparys, Saint Cloud, F-92210, France \\ ${ }^{5} \mathrm{CHU}$ Rennes, Service de Cardiologie et Maladies Vasculaires, Rennes, F-35000, France
}

\begin{abstract}
Heart sounds, measured via cardiac micro-acceleration (CMA), carry valuable information about the mechanical and hemodynamic function of the heart. The aim of the present study is to estimate systolic and diastolic time intervals from CMA features, extracted by segmentation of the first two heart sounds, i.e. onset, maximum energy and end instants. CMA, ECG and echocardiographic audio signals were acquired simultaneously on 60 patients under different hemodynamic conditions. Linear models, fitted between CMA features and echo timings, were evaluated with their correlation coefficient, model error and coefficient stability. Models for mitral valve closing, aortic valve closing and opening instants showed satisfactory results, whereas the estimation of the opening instant of the mitral valve was more difficult. This work suggests the potential utility of CMA for monitoring cardiac function and defining optimal, adaptive pacing configurations.
\end{abstract}

\section{Introduction}

Cardiac Resynchronization Therapy (CRT) is indicated for patients suffering from drug-refractory congestive heart failure (CHF) associated with inter-ventricular dyssynchrony [1]. The implantation of a bi-ventricular stimulator makes it possible to re-synchronize the electrical activation of both ventricles. In order to maximize the cardiac mechanical function for a given patient, the atrio-ventricular (AV) and inter-ventricular (VV) activation delays of the device have to be optimized.

This optimization process is a difficult task that often involves an echo-Doppler acquisition, in order to evaluate the ventricular mechanical function, while scanning different values for AV and VV delays. This cumbersome technique is not applied systematically and is only done at rest, in supine position. Instead, the possibility of using a car- diac acoustic signal to monitor the mechanical function of the heart would be interesting from several points of view: i) it will ease the application of the optimization stage, reducing costs, ii) it will allow the evaluation on different conditions (rest, exercise, etc.), and iii) it could eventually be embedded into a pacemaker in order to perform an automatic and adaptive CRT optimization.

The study of cardiac acoustic signals has shown to be useful for the evaluation of the mechanical function of the heart. Methods for the analysis of the phonocardiogram (PCG) or the seismocardiogram (SCG) have been proposed to extract useful information and to perform heart sounds segmentation [2-5]. The arrival of Doppler echocardiography significantly reduced the clinical use of these signals, but the precision and reproducibility of echoDoppler measurements have been repeatedly criticized [6]. This fact might lead to a renewed interest in quantitative analysis of cardiac acoustic signals, especially in the field of CRT [7].

In this context, previous studies have shown that the measurement of heart sounds via endocardial acceleration (EA) (see Fig 1), developed by Sorin Group CRM, may be valuable for an online follow-up of the cardiac mechanical function. In particular, the peak to peak amplitude of first component of the EA signal (EA1) is well correlated to the maximum rate of rise of left ventricular pressure [8] and has been successfully used to optimize CRT [1].

The present study focuses on a non-invasive cardiac micro-acceleration (CMA) device, based on an external version of the EA sensor, placed on the chest of the patient. The potential use of CMA in the context of CRT is evaluated by a quantitative comparison between extracted CMA timings and Doppler-based time intervals. An evaluation methodology is proposed to assess the performance of different heart sounds segmentation algorithms. 


\section{Methods}

\subsection{Protocol}

This study is based on a non-interventional multi-centre, prospective registry, including $51 \mathrm{CHF}$ patients, and 9 healthy subjects.

Each patient recording was collected during a routine follow-up including echocardiographic measurements. 51 heart failure patients, with implanted CRT pacemakers have been studied. In order to change the systolic and diastolic time intervals, different pacing configurations were tested: i) biventricular pacing, ii) right ventricular (RV) pacing, iii) left ventricular (LV) pacing, and, when possible, iv) spontaneous rhythm. For some patients, different $\mathrm{AV}$ and VV delays were tested.

Echo stress tests were performed on 9 healthy volunteers at rest, during exercise (cycling), and during recovery. These subjects are important, because focusing on CRT patients only would lead to a limited exploration of the possible values of the studied parameters. For example, healthy subjects show shorter left pre-ejection interval (LPEI) values and shorter cardiac cycles during controlled exercise.

For each configuration, a simultaneous recording of CMA, 2-Lead ECG and pulsed-wave Doppler audio signal, taken from the audio output of a GE Vivid7, were acquired with a Biopac MP35 acquisition system at $10 \mathrm{kHz}$. The CMA sensor was clipped with a standard surface ECG electrode on the sternum of the patient and analogically filtered in the $[15-100 \mathrm{~Hz}]$ range. Data was processed off-line with custom Matlab signal processing routines.

\subsection{Gold standard Doppler measurements}

ECG signals were used to segment CMA and Doppler signals into cardiac cycles. We performed an automatic beat detection from ECG signals, and then manually verified and corrected the results. This first step, yet simple, is a real improvement regarding the method used in every day clinical practice. We attached much importance to the accuracy and reproducibility of the Doppler measurements. Mitral and aortic blood flow were reconstructed from the Doppler audio signal by applying short-time FFT, with a Hamming window of 64 samples (Fig. 1). To ease the annotations, a flow envelogram was estimated by first integrating the Doppler velocities at each time sample and then applying a moving-average filter.

Four valvular events were annotated by a trained operator and validated by an expert echocardiographist: i) $t M C$ $=t R E F$ to mitral valve closing, ii) $t A O(\mathrm{LPEI})=t R E F$ to aortic valve opening, iii) $t A C=t R E F$ to aortic valve closing, iv) $t M O=t R E F$ to mitral valve opening, where $t R E F$ is either the beginning of the pacing spike or the be-
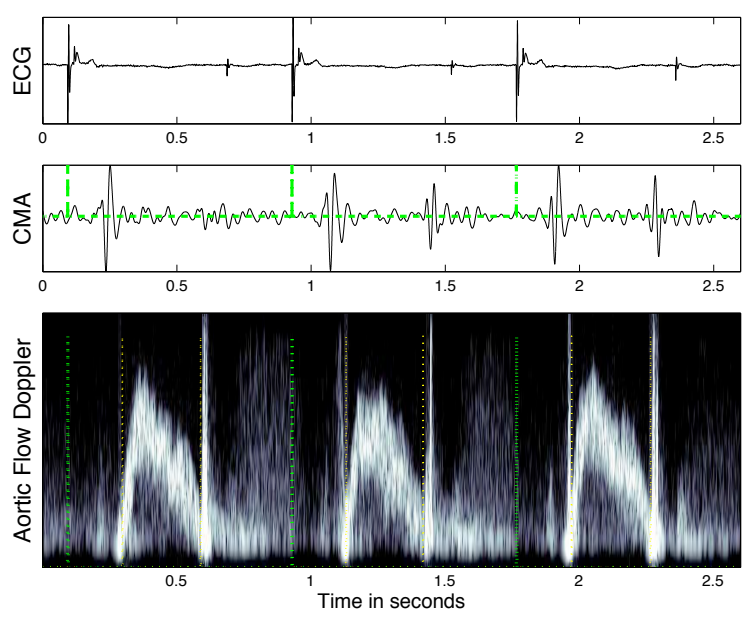

Figure 1. ECG, CMA and aortic flow Doppler. The beginning of each beat, the opening and closing of the aortic valve appear as dotted lines on the lower panel.

ginning of the $\mathrm{Q}$ wave in spontaneous rhythms. When the echo measurements confidence on a segment was judged too low by the echocardiographist, the segment of data was rejected from the database.

\subsection{Extraction of CMA timings}

For each configuration, 15 cycles of CMA signal, occurring during the acquisition of the pulsed wave Doppler, are considered for standard ensemble averaging of the two main components of the CMA signal, EA1 and EA2 (corresponding respectively to the first and second heart sound in the PCG), separately: i) the phase shifts that maximize the correlation between each cycle are calculated, ii) the cycles are aligned according to a reference component (first cycle of the analysis window) and iii) the two average components EA1 and EA2 are computed. Only highly correlated cycles are averaged.

Envelograms are computed on this average CMA cycle, by applying both the Shannon and Homomorphic transforms. These transforms have already been applied to the segmentation of PCG signals $[3,4]$.

Finally, a threshold-based segmentation algorithm is applied to the envelograms. Again, EA1 and EA2 envelograms are normalized and processed separately, in order to estimate their start and end instants $\left(t_{E \text { AxStart }}\right.$ and $t_{E A x E n d}$ respectively) and their instant of maximum energy $\left(t_{E A x_{\text {max }} \text { energy }}\right)$. Scanning different values of $w$ (defined as the duration of the moving-average window for the Shannon transform and the low-pass filter cut-off frequency for Homomorphic envelograms) and threshold (from 0.1 to 0.7 by steps of 0.1 ) generates different CMA instants. 


\subsection{Performance assessment}

We first removed outlier points (typically outside the $30 \mathrm{~ms}$ model error boundaries). A linear model $(t V .=$ $a \cdot$ feat $+b$, where $t V$. is the reference event for valve $V$ and feat is a CMA feature) was then estimated by standard RMSE minimization. Two complementary approaches were used to evaluate each model:

- Single-pass: a single model is estimated per feature on $N$ points (all patients and configurations included). This model is globally evaluated with its correlation coefficient $r_{L}$, its average absolute error $\mu_{e r r}$, and the percentage of points outside the $20 \mathrm{~ms}$ error limit \%out.

- Monte-Carlo: on the entire database (DB), we generate 27 different pairs of learning DB (60\% of the DB), on which the model is estimated, and test DB (40\% of the DB), on which the model is evaluated. In each case, $r_{T}$ and the model error are calculated. We can then estimate the average correlation coefficient $\mu_{r_{T}}$ and the stability of the models for each feature: the standard deviation of each model's coefficient ( $a$ and $b$ ) is calculated $\left(\sigma_{a}\right.$ and $\sigma_{b}$ ).

\section{Results and discussion}

The best CMA feature for each one of the 4 reference echo timings are detailed in Table 1, with their corresponding optimal segmentation parameters. It is worth to note that these optimal parameters vary for different echo timings, even if the same CMA feature is selected. For $t A O$, we specified 2 different CMA features because they provide similar performance.

- Valves Closing Times $(t M C$ and $t A C)$ : $t M C$ can be estimated accurately from several $t_{E A 1 \text { Start }}$ features : $\mu_{\text {err }}=12 \mathrm{~ms}, r_{L}=0.89$ and \%out $=16$. Similarly, several $t_{E A 2 S t a r t}$ timings are good estimators of $t A C$ : $\mu_{\text {err }}=9 \mathrm{~ms}, r_{L}=0.98$ and \%out $=7$. Figure 2 presents an example of the estimation of $t A C$ from $t_{E A 2 S t a r t}$ by using a Homomorphic envelogram with $w=15 \mathrm{~Hz}$ and threshold $=0.6$.

Moreover, these models are very stable: $\sigma_{a}$ values for $t M C$ models are relatively low (2.6\% variations of their corresponding $a$ value) and for $t A C$ models ( $0.6 \%$ variations). The same stability is observed on the $b$ coefficients for $t M C$ and $t A C$ models $\left(\sigma_{b}=3 \mathrm{~ms}\right)$.

- Valves Opening Times ( $t M O$ and $t A O)$ : a satisfactory estimation of the absolute value of $t A O$ with the present extracted CMA timings was difficult to obtain, in comparison with those obtained for valve closing instants, but the best model involving a $t_{E A 1 \text { Start }}$ feature presents overall interesting properties: $\mu_{e r r}=17 \mathrm{~ms}, r_{L}=0.86$ and $\%$ out $=35$. This can be explained by the fact that several complex physiopathological phenomena (including $t M C$ and $t A O$ ) are at the origin of EA1 (as for the first heart sound in the PCG) and their relative time of occurrence may vary in an intra and inter-patients fashion. However, a good correlation exists between $t_{E A 1 \text { Start }}$ (see Fig. 3) or $t_{E A 1_{\text {max }} \text { energy }}$ and the Doppler LPEI, as shown in Table 1. Concerning $t M O$, we are not currently able to locate this particular time instant in the CMA signal. In this case, the presence of healthy subjects at rest and performing exercise has a great influence on the correlation $r_{L}=0.9$, despite a high model error $\mu_{\text {err }}=27 \mathrm{~ms}$ and a significant proportion of patients out of the $20 \mathrm{~ms}$ error boundaries \%out $=62$.

The Monte Carlo analysis demonstrates that these models are more unstable, especially on $t M O . \sigma_{a}$ are high for $t A O$ models (8\% variations of their respective $a$ value for $t_{E A 1 \text { Start }}$ and $11 \%$ for $t_{E A 1_{\text {maxenergy }}}$ ) and $t M O$ models ( $8 \%$ variations). This is particularly true for the $b$ coefficients on $t A O\left(\sigma_{b}=14 \mathrm{~ms}\right.$ for $t_{E A 1 S t a r t}$ and $21 \mathrm{~ms}$ for $\left.t_{E A 1_{\text {max }} \text { energy }}\right)$ and $t M O$ models $\left(\sigma_{b}=46 \mathrm{~ms}\right)$.

It should be observed that $t_{E A 1 S t a r t}$ features correlate well to both $t A O$ and $t M C$, despite the lack of a significant correlation between these two Doppler instants $(r=0.59)$. This may be due to the fact that i) $t_{E A 1 S t a r t}$ detection instants are more stable than those of $t_{E A 1_{\text {max }} \text { energy }}$ (respectively $\sigma_{a}=0.094$ and 0.118 ) and ii) the delay separating $t M C$ and $t A O$ (the isovolumic contraction time) is absorbed by the model's coefficients $(b=37 \mathrm{~ms}$ for $t A O$ and $5 \mathrm{~ms}$ for $t M C$ ). This phenomenon is similar to the one observed on $t A C$ (small model error) and $t M O$ (large model error), except that here the two Doppler instants are significantly correlated $(r=0.87)$.

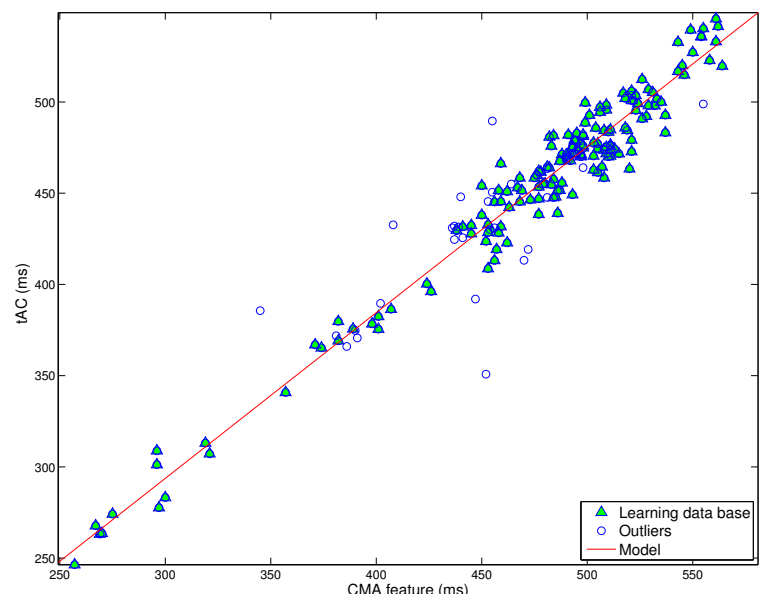

Figure 2. $t A C$ model estimation from $t_{E A 2 S t a r t}$

\subsection{Conclusion}

Results clearly demonstrate the feasibility of estimating the mitral and aortic valve closing instants from cardiac micro-acceleration features and suggest their potential application in a clinical context. Regarding $t A O$, we 
Table 1. Model performances

\begin{tabular}{|c||c|c|c|c|ccccccccc}
\hline & CMA feature & Method & $w$ & thres. & $N$ & $\mu_{\text {err }}$ & $\sigma_{\text {err }}$ & $r_{L}$ & $\%$ out & $\mu_{r_{T}}$ & $\sigma_{a}$ & $\sigma_{b}$ \\
\hline \hline$t M C$ & $t_{E A 1 S t a r t}$ & Shannon & $40 \mathrm{~ms}$ & 0.4 & 172 & $12 \mathrm{~ms}$ & $8 \mathrm{~ms}$ & 0.89 & 16 & 0.88 & 0.025 & 0.003 \\
\hline$t A O$ & $t_{E A 1 S t a r t}$ & Shannon & $80 \mathrm{~ms}$ & 0.6 & 170 & $17 \mathrm{~ms}$ & $13 \mathrm{~ms}$ & 0.86 & 35 & 0.81 & 0.094 & 0.014 \\
\hline$t A O$ & $t_{E A 1_{\text {max }} \text { energy }}$ & Shannon & $150 \mathrm{~ms}$ & - & 170 & $17 \mathrm{~ms}$ & $14 \mathrm{~ms}$ & 0.83 & 37 & 0.78 & 0.118 & 0.021 \\
\hline$t A C$ & $t_{E A 2 \text { Start }}$ & Homomorphic & $15 \mathrm{~Hz}$ & 0.6 & 172 & $9 \mathrm{~ms}$ & $7 \mathrm{~ms}$ & 0.98 & 7 & 0.96 & 0.006 & 0.003 \\
\hline$t M O$ & $t_{E A 2 \text { Start }}$ & Shannon & $80 \mathrm{~ms}$ & 0.2 & 182 & $27 \mathrm{~ms}$ & $17 \mathrm{~ms}$ & 0.9 & 62 & 0.83 & 0.098 & 0.046 \\
\hline
\end{tabular}

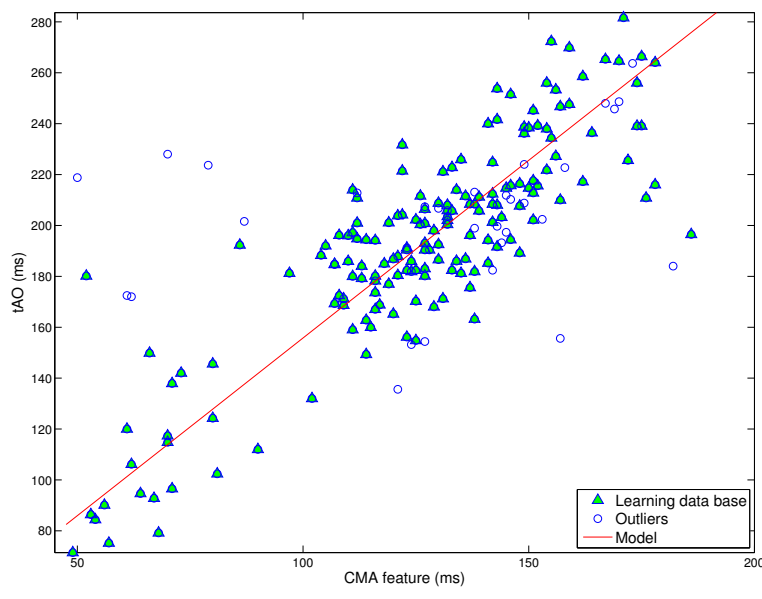

Figure 3. $t A O$ model estimation from $t_{E A 1 S t a r t}$

obtained suitable models for two different types of EA1 features. Ongoing work is directed to the improvement of the estimation of mitral valve opening instant. The proposed algorithms showed to be robust with respect to environmental noise and artifacts, usually present on this kind of surface acoustic signals. It would now be relevant to evaluate the stability of the CMA timings during a given hemodynamic configuration, on successive groups of cardiac cycles.

We chose echocardiographic measurements as a gold standard, despite its drawbacks, (i.e. operator dependency, position of the transducer, limited number of beats), because it is still recognized by the physician community as a reference method. These limitations have to be kept in mind, even though we significantly enhanced the reliability of this reference with specific signal processing tools.

To conclude with, the clinical potential of these CMA features seems really promising, and, combined with appropriate implantable sensors and stimulators, could set the grounds for a significant advance in CRT.

\section{Acknowledgements}

This work was supported by Sorin Group CRM.The autors gratefully acknowledge the assistance of P. Lotton in CHU Rennes, M. Heurteau and L. Graindorge from Sorin Group for their support for data collection.

\section{References}

[1] Dupuis JM, Kobeissi A, Vitali L, Gaggini G, Merheb M, Rouleau F, Leftheriotis G, Ritter P, Victor J. Programming optimal atrioventricular delay in dual chamber pacing using peak endocardial acceleration: comparison with a standard echocardiographic procedure. Pacing Clin Electrophysiol Jan 2003;26(1 Pt 2):210-213.

[2] Durand LG, Pibarot P. Digital signal processing of the phonocardiogram: review of the most recent advancements. Crit Rev Biomed Eng 1995;23(3-4):163-219.

[3] Liang H, Lukkarinen S, Hartimo I. Heart sound segmentation algorithm based on heart sound envelogram. In Proc. Computers in Cardiology 1997. 7-10 Sept. 1997; 105-108.

[4] Gill D, Gavrieli N, Intrator N. Detection and identification of heart sounds using homomorphic envelogram and selforganizing probabilistic model. In Proc. Computers in Cardiology. Sept. 25-28, 2005; 957-960.

[5] Marcus FI, Sorrell V, Zanetti J, Bosnos M, Baweja G, Perlick D, Ott P, Indik J, He DS, Gear K. Accelerometer-derived time intervals during various pacing modes in patients with biventricular pacemakers: comparison with normals. Pacing Clin Electrophysiol Dec 2007;30(12):1476-1481.

[6] Chung ES, Leon AR, Tavazzi L, Sun JP, Nihoyannopoulos P, Merlino J, Abraham WT, Ghio S, Leclercq C, Bax JJ, Yu CM, Gorcsan J, Sutton MSJ, Sutter JD, Murillo J. Results of the predictors of response to crt (prospect) trial. Circulation May 2008;117(20):2608-2616.

[7] Zuber M, Toggweiler S, Quinn-Tate L, Brown L, Amkieh A, Erne P. A comparison of acoustic cardiography and echocardiography for optimizing pacemaker settings in cardiac resynchronization therapy. Pacing Clin Electrophysiol Jul 2008;31(7):802-811.

[8] Plicchi G, Marcelli E, Parlapiano M, Bombardini T. Pea i and pea ii based implantable haemodynamic monitor: pre clinical studies in sheep. Europace Jan 2002;4(1):49-54.

Address for correspondence:

Lionel Giorgis

LTSI - Université de Rennes 1 - Campus de Beaulieu - Bat. 22 -

35042 RENNES - FRANCE - lionel.giorgis@ sorin.com 